\title{
In memoriam Arnold Picot
}

\author{
Marina Fiedler
}

Online publiziert: 24. Juli 2017

(C) Schmalenbach-Gesellschaft für Betriebswirtschaft e.V. 2017

Arnold Picot ist am Sonntag, den 09.07.2017, plötzlich und unerwartet im Alter von 72 Jahren gestorben. Er kann auf zahlreiche Verdienste in einer über 45-Jahre andauernden großartigen Karriere zurückblicken. Die Schmalenbach Business Review und die Zeitschrift für betriebswirtschaftliche Forschung trauern um ihren Mitherausgeber, der Schriftführer des Kontaktstudiums war und sich frühzeitig und engagiert für die Schmalenbach Business Review als englischsprachige Ausgabe einsetzte.

Arnold Picot war für viele Jahre einer der wichtigsten Wissenschaftler und Vordenker der Betriebswirtschaft in Deutschland. Inhaltlich wurde er vor allem dafür bekannt, dass er mit Hilfe der neuen Institutionenökonomie half, den Nutzen und die Grenzen von neuen Technologien besser zu verstehen - und zu analysieren wie sich dadurch Organisationen, Branchen und Märkte verändern. Die so entwickelten Erkenntnisse, Befunde und Regeln waren dabei oft der Umsetzung weit voraus, was ihn zu einem begehrten Gesprächspartner in Wissenschaft, Politik und Praxis machte. So übernahm er seinen ersten Lehrstuhl bereits 1976 an der Universität Hannover bevor er 1984 einem Ruf der TU München und 1988 der Ludwig-Maximilians-Universität folgte, an der er auch nach seiner Pensionierung mehrere Forschungsprojekte durchführte. Er war bei der Deutschen Forschungsgemeinschaft seit über 25 Jahren als Gutachter, Mitglied im Senat und Hauptausschuss und als Vertrauensdozent für die LMU tätig. Er war zudem über 18 Jahre Vorsitzender des wissenschaftlichen Arbeitskreises für Regulierungsfragen bei der Bundesnetzagentur und über 14 Jahre Vorstandsvorsitzender des MÜNCHNER KREIS. Als Mitglied der acatech wirkte er bis zuletzt in verschiedenen Initiativen mit.

\footnotetext{
M. Fiedler $(\triangle)$

Lehrstuhl für Management, Personal und Information (LMPI)/Chair for Management, People and Information, Universität Passau I Wirtschaftswissenschaftliche Fakultät/ School of Business Administration and Economics, Innstr. 27, 94032 Passau, Deutschland

E-Mail: marina.fiedler@uni-passau.de
} 
Dass er so vielen verschiedenen Interessengruppen mit seiner Expertise zur Seite stand, lag vor allem daran, dass Arnold Picot über die Fähigkeit zukunftsgerichteten Denkens verfügte. Eine Frage aus dem Kontext heraus zu verstehen, neue Inhalte in diesen einzubetten, überdurchschnittlich schnell $\mathrm{zu}$ erkennen, was wichtig und relevant ist und damit die Komplexität besser zu bewältigen, darin war er außerordentlich gut. Wenn man von ihm hörte: „Das musst Du mir nochmal genauer erklären" wusste man, dass sich die eigene Argumentation noch auf dünnem Eis bewegte.

Geholfen hat ihm dabei, dass er die BWL nicht ausschließlich als empirische, sondern auch als konstruktive Wissenschaft verstand. Gute Wissenschaft zeichnete sich für ihn auch immer dadurch aus, dass man sich selbst treu bleibt, an seine eigenen Fähigkeiten und Ideen glaubt und die vielfältigen Möglichkeiten des Berufs als Professor nutzt, um das Arbeitsfeld gemäß eigenen Interessen und dadurch mit Freude zu gestalten. Die Praxis verstand er dabei als wichtigen, komplementären Faktor, um die Relevanz und die Validität dessen, was in der Wissenschaft passiert, zu erproben. So war er u. a. seit 1998 bis zuletzt Aufsichtsratsvorsitzender der Sartorius AG.

Dabei war es ihm von Anfang an wichtig, im Team mit interessanten und herausfordernden Menschen zusammenzuarbeiten und über eine Vielfalt von Gremien in die Gesellschaft hineinzuwirken. So betreute er über 100 eigene Schüler, fungierte als Vizepräsident der bayerischen Akademie der Wissenschaften, wirkte als Mitglied des Wissenschaftlichen Beirats des Zentrums für Europäische Wirtschaftsforschung Mannheim und saß seit 2003 bis zuletzt dem Hochschulrat der Leibniz Universität Hannover vor.

Er war stets offen für die verschiedensten Argumente und immer für eine Diskussion auf Augenhöhe bereit. Bei vielen seiner Weggefährten war er deshalb nicht nur für seine fachliche Leistung geschätzt, sondern für seine zutiefst integrative Art, mit der er der Welt begegnete und Brücken zwischen verschiedenen Positionen und Menschen baute. Er ging mit den ihn umgebenden Personen immer ermunternd, sozial intelligent und verantwortungsvoll um und hat damit viel bewirkt, um größere Ziele zu erreichen. Dabei blieb er trotz zahlreicher Ehrungen und Anerkennungen stets bescheiden. So war er beispielsweise Ehrenmitglied des Verbands der Hochschullehrer für Betriebswirtschaft und erhielt die Ehrendoktorwürden der TU Bergakademie Freiberg sowie der Universität St. Gallen, verweigerte sich aber zum Beispiel einer offiziellen Abschiedsvorlesung und Festschrift anlässlich seiner Pensionierung. Das führte dazu, dass seine Familie, Kollegen und Teammitglieder letztendlich mit Rasseln und Tröten in seine letzte reguläre Vorlesung kamen, um doch irgendwie eine Art von - wenn auch unkonventioneller - Wertschätzung zu zeigen.

Dass ausgerechnet er, der so vielen Menschen mit klugem Rat und Menschlichkeit zur Seite stand, so plötzlich und früh aus dem Leben gerissen wurde, ist tragisch. Tröstlich ist dabei nur, dass er als glücklicher Mensch von uns gegangen ist, der ein erfülltes, sinnvolles Leben geführt hat und sich immer der Vergänglichkeit von Lebenszeit bewusst war. Seine Ideen und Perspektiven werden in den zukünftigen Aktivitäten der Schmalenbach Gesellschaft, in der er bis zuletzt im Vorstand aktiv war, weiter wirken. 